\title{
MEROMORPHIC SOLUTIONS OF A SYSTEM OF FUNCTIONAL EQUATIONS INVOLVING THE MODULAR GROUP1
}

\author{
MICHAEL M. BRADY ${ }^{2}$
}

Abstract. The purpose of this paper is to study the meromorphic solutions $F$ of the system of functional equations (1) $F[T(z)]$ $=T[F(z)]$, for all $T$ in the modular group $M$ and $z$ in the upperhalf plane. A formula is given which expresses all such solutions explicitly in terms of the functions automorphic relative to $M$. Then, an application of the functions that satisfy (1) is given, and some observations are made concerning solutions of the more general system of functional equations that results when $M$ is replaced by an arbitrary Fuchsian group.

1. Introduction. Let $U$ denote the upper-half plane $U$ $=\{z: \operatorname{Im} z>0\}$, and let $M$ denote the classical modular group which consists of the linear fractional transformations

$$
T(z)=(a z+b) /(c z+d),
$$

$a, b, c, d$ being real integers satisfying $a d-b c=1$. The purpose of this paper is to study the solutions $F$ meromorphic on $U$ of the system of functional equations

$$
F[T(z)]=T[F(z)],
$$

for all $T \in M, z \in U$. Let $S$ denote the class of all such functions. The identity function restricted to $U$ is a member of $\boldsymbol{S}$, and $\mathbf{M}$. Heins showed [3] that all other members of $S$ have $U$ as their natural boundary and take on every value of the extended complex plane $\hat{C}$ at infinitely many points mutually nonequivalent relative to $M$. Heins also exhibited a member of $S$ that is not the identity function. In this paper a formula is given that expresses all the members of $\mathcal{S}$ explicitly in terms of the functions automorphic relative to $M$. It

Received by the editors August 24, 1970.

AMS 1969 subject classifications. Primary 3060, 3049; Secondary 3319.

Key words and phrases. Functional equations, modular group, automorphic functions, pseudoperiodic functions, Fuchsian group, Weierstrass zeta-function.

1 This research contains part of the author's doctoral dissertation written under the supervision of Professor Maurice H. Heins at the University of Illinois, Urbana.

2 This research was supported by NSF Grant No. GP-7405, Center for Naval Analyses, Washington, D. C., and Esso Production Research Company, Houston, Texas. 
will follow that $S$ has the power of the continuum. We then give an application of the functions in $\delta$ by using their mapping properties to solve a set of equations from the theory of pseudoperiodic functions. In the last section some observations are made concerning meromorphic solutions of the more general system of functional equations that results when $M$ is replaced by an arbitrary Fuchsian group $G$. For this general case, it is shown that if there exists three mutually distinct meromorphic solutions of (1), then all the meromorphic solutions of (1) can be expressed explicitly in terms of the functions automorphic relative to $G$. The existence, or nonexistence, of three mutually distinct solutions appears to be unsettled.

2. Preliminaries. In this paragraph we recall some basic notions. For $\omega_{1}, \omega_{2}$ in the complex plane $C$ such that $\operatorname{Im} \omega_{1}^{-1} \omega_{2} \neq 0$, let $\Omega\left(\omega_{1}, \omega_{2}\right)$ denote the subgroup of $C$, considered additively, generated by $\omega_{1}$ and $\omega_{2}$. Let $\mathscr{L}$ denote the set of all such $\Omega\left(\omega_{1}, \omega_{2}\right)$. Let $f: C \times \mathscr{L} \rightarrow \hat{C}$ be a meromorphic function of $z$ on $C$ for fixed $\Omega \in \mathcal{L}$. $f$ is said to be pseudoperiodic if for each $\omega \in \Omega$, there exists a constant $H(\omega \mid \Omega) \in \hat{C}$ such that

$$
f(z+\omega \mid \Omega)=f(z \mid \Omega)+H(\omega \mid \Omega),
$$

for all $z \in C$. An important property of the pseudoperiods, $H(\omega \mid \Omega)$, is that they are linear in the first argument, i.e., $H\left(m \omega_{1}+n \omega_{2} \mid \Omega\right)$ $=m H\left(\omega_{1} \mid \Omega\right)+n H\left(\omega_{2} \mid \Omega\right)$, for $m \omega_{1}+n \omega_{2} \in \Omega$. $f$ is said to be homogeneous of weight $\mu, \mu$ a real integer, if $f(\lambda z \mid \lambda \Omega)=\lambda^{\mu} f(z \mid \Omega)$, for $\lambda \in C-\{0\}$. Here $\lambda \Omega\left(\omega_{1}, \omega_{2}\right)=\Omega\left(\lambda \omega_{1}, \lambda \omega_{2}\right)$. It follows that if $f$ is homogeneous of weight $\mu$, then so are the pseudoperiods, i.e., $H(\lambda \omega \mid \lambda \Omega)=\lambda^{\mu} H(\omega \mid \Omega)$. An important homogeneous $(\mu=-1)$ pseudoperiodic function is the Weierstrass zeta-function $\zeta: C \times \mathfrak{L} \rightarrow \hat{C}$ defined by

$$
\zeta(z \mid \Omega)=\frac{1}{z}+\sum_{\omega \in \Omega ; \omega \neq 0}\left[\frac{1}{z-\omega}+\frac{1}{\omega}+\frac{z}{\omega^{2}}\right] .
$$

It is customary to denote the pseudoperiods of $\zeta$ by $\eta(\omega \mid \Omega)$. If $\Omega=\Omega\left(\omega_{1}, \omega_{2}\right)$, then $\omega_{1}$ and $\omega_{2}$ can always be chosen so that $\operatorname{Im} \omega_{1}^{-1} \omega_{2}>0$, and in this case we have Legendre's relation [5]

$$
\eta\left(\omega_{1} \mid \Omega\right) \omega_{2}-\eta\left(\omega_{2} \mid \Omega\right) \omega_{1}=2 \pi i .
$$

As a consequence of (2) it follows that every pseudoperiodic function $f$ can be written uniquely in the form

$$
f(z \mid \Omega)=a(\Omega) z+b(\Omega) \zeta(z \mid \Omega)+E(z \mid \Omega) .
$$

Here $a(\Omega), b(\Omega) \in C$, and $E$ is an elliptic function, i.e., $E(z+\omega \mid \Omega)$ $=E(z \mid \Omega)$ for $\omega \in \Omega, z \in C$. The pseudoperiods $H(\omega \mid \Omega)$ of $f$ are the form 


$$
H(\omega \mid \Omega)=a(\Omega) \omega+b(\Omega) \eta(\omega \mid \Omega) .
$$

A function $A$ meromorphic on $U$ is said to be automorphic relative to $M$ if $A[T(z)]=A(z)$, for all $T \in M, z \in U$. Let $\propto$ be the class of all such functions. The functions in $Q$ are also called modular functions. The classical modular function $J$ is defined as

$$
J(\tau)=\frac{g_{2}^{3}\left(\Omega_{\tau}\right)}{g_{2}^{3}\left(\Omega_{\tau}\right)-27 g_{3}^{2}\left(\Omega_{\tau}\right)},
$$

for $\tau \in U$. Here $\Omega_{\tau}=\Omega(1, \tau)$ and $g_{i}\left(\Omega_{\tau}\right)=\hat{g}_{i}\left(\Omega_{\tau}\right), i=1,2$, where $\hat{g}_{i}: \mathcal{L} \rightarrow C$ are the invariants $\hat{g}_{2}(\Omega)=60 \quad \sum_{\omega \in \Omega ; \omega \neq 0}\left(1 / \omega^{4}\right)$ and $\hat{g}_{3}(\Omega)$ $=140 \sum_{\omega \in \Omega ; \omega \neq 0}\left(1 / \omega^{6}\right)$ from elliptic function theory [5]. $g_{2}$ and $g_{3}$ are analytic functions of $\tau$ on $U$ and $g_{2}^{3}\left(\Omega_{\tau}\right)-27 g_{3}^{2}\left(\Omega_{\tau}\right) \neq 0$, which implies that $J$ is analytic on $U$. We note that for every automorphic function $A: U \rightarrow \hat{C}$, a function $\hat{A}: \mathfrak{L} \rightarrow \hat{C}$ can be defined such that $A(\tau)=\hat{A}\left(\Omega_{\tau}\right)$. Let $\hat{A}(\Omega)=A\left(\omega_{2} / \omega_{1}\right)$, where $\Omega=\Omega\left(\omega_{1}, \omega_{2}\right)$ and $\operatorname{Im} \omega_{1}^{-1} \omega_{2}>0$. $\hat{A}$ is well-defined since if $\Omega\left(\omega_{1}, \omega_{2}\right)=\Omega\left(\omega_{1}^{\prime}, \omega_{2}^{\prime}\right)$ and $\operatorname{Im} \omega_{1}^{-1} \omega_{2}, \operatorname{Im} \omega_{1}^{\prime-1} \omega_{2}^{\prime}$ $>0$, then $\omega_{2}^{\prime} / \omega_{1}^{\prime}=T\left(\omega_{2} / \omega_{1}\right)$ for some $T \in M[5]$.

\section{Main theorem.}

Lemma. Let $f: C \times \mathfrak{L} \rightarrow \hat{C}$ be a pseudoperiodic function with pseudoperiods $H(\omega \mid \Omega)$ such that (a) $f$ is homogeneous,

(b) $\tau \rightarrow H\left(1 \mid \Omega_{\tau}\right)$ and $\tau \rightarrow H\left(\tau \mid \Omega_{\tau}\right)$ are meromorphic functions of $\tau$ on $U$ and $H\left(1 \mid \Omega_{\tau}\right) \not \equiv 0$.

Then the function $F$ defined by $F(\tau)=H\left(\tau \mid \Omega_{\tau}\right) / H\left(1 \mid \Omega_{\tau}\right)$ is in $\mathrm{S}$.

Proof. It is an immediate consequence of (b) that $F$ is meromorphic on $U$. Since the subgroup of $C$, considered additively, generated by $\{a \tau+b, c \tau+d\}$ is the same as that generated by $\{1, \tau\}$, for $T(z)$ $=(a z+b) /(c z+d) \in M$, we observe that $\Omega_{T(\tau)}=1 /(c \tau+d) \Omega_{\tau}$. When $H\left(1 \mid \Omega_{\tau}\right) \neq 0, H\left(1 \mid \Omega_{\tau}\right) \neq \infty, H\left(z \mid \Omega_{\tau}\right) \neq \infty$, we have

$$
\begin{aligned}
T[F(\tau)] & =\frac{\left(a \frac{H\left(\tau \mid \Omega_{\tau}\right)}{H\left(1 \mid \Omega_{\tau}\right)}+b\right)}{\left(c \frac{H\left(\tau \mid \Omega_{\tau}\right)}{H\left(1 \mid \Omega_{\tau}\right)}+d\right)}=\frac{a H\left(\tau \mid \Omega_{\tau}\right)+b H\left(1 \mid \Omega_{\tau}\right)}{c H\left(\tau \mid \Omega_{\tau}\right)+d H\left(1 \mid \Omega_{\tau}\right)} \\
& =\frac{H\left(a \tau+b \mid \Omega_{\tau}\right)}{H\left(c \tau+d \mid \Omega_{\tau}\right)}=\frac{H\left[a \tau+b \mid(c \tau+d) \Omega_{T(\tau)}\right]}{H\left[c \tau+d \mid(c \tau+d) \Omega_{T(\tau)}\right]} \\
& =\frac{(c \tau+d)^{\mu} H\left[T(\tau) \mid \Omega_{T(\tau)}\right]}{(c \tau+d)^{\mu} H\left[1 \mid \Omega_{T(\tau)}\right]}=F[T(\tau)] .
\end{aligned}
$$


Since the set of points where $H\left(1 \mid \Omega_{\tau}\right)=0, H\left(1 \mid \Omega_{\tau}\right)=\infty$, or $H\left(\tau \mid \Omega_{\tau}\right)$ $=\infty$ is isolated, $T[F(\tau)]-F[T(\tau)]=0$ for all $\tau \in U$ by the identity theorem for meromorphic functions. Q.E.D.

The identity function $I: C \times \& \rightarrow \hat{C}$ given by $I(z \mid \Omega)=z$ satisfies (a) and (b) and $F(\tau)=H\left(\tau \mid \Omega_{\tau}\right) / H\left(1 \mid \Omega_{\tau}\right)=\tau / 1=\tau$. The Weierstrass zetafunction $\zeta$ also satisfies (a) and (b) and $F(\tau)=\eta\left(\tau \mid \Omega_{\tau}\right) / \eta\left(1 \mid \Omega_{\tau}\right)$ is the function that Heins exhibited in [3]. Let $\eta_{1}, \eta_{2}$, and $I_{U}$ denote the functions $\tau \rightarrow \eta\left(1 \mid \Omega_{\tau}\right), \tau \rightarrow \eta\left(\tau \mid \Omega_{\tau}\right)$, and the identity function restricted to $U$, respectively.

Theorem 1. The map $\Phi: C \times a-\{0,0\} \rightarrow \delta$ defined by

$$
\Phi(a, A)=\left(a g_{3} I_{U}+g_{2} A \eta_{2}\right) /\left(a g_{3}+g_{2} A \eta_{1}\right)
$$

is onto $S$, and $\Phi(a, A)=\Phi(b, B)$ if and only if $\left|\begin{array}{ll}a & b \\ A & B\end{array}\right|=0$.

Proof. Consider the pseudoperiodic function $f$ defined by

$$
(z, \Omega) \stackrel{f}{\rightarrow} a \hat{g}_{3}(\Omega) z+\hat{g}_{2}(\Omega) \hat{A}(\Omega) \zeta(z \mid \Omega) .
$$

It is easily verified that $f$ satisfies (a) and (b). Therefore, $\Phi(a, A) \in \delta$ by the Lemma. We show that $\Phi(a, A)=\Phi(b, B)$ if and only if $\left|\begin{array}{cc}a & b \\ A & B\end{array}\right|=0$. Suppose that $\Phi(a, A)=\Phi(b, B)$. Then after cross-multiplying and cancelling we have $g_{2} g_{3}(a B-b A) \eta_{2}=g_{2} g_{3}(a B-b A) \eta_{1} I_{U}$. It follows that $\left|\begin{array}{cc}a & b \\ A & B\end{array}\right|=0$, since otherwise Legendre's relation (2) would be contradicted for some $(1, \tau), \tau \in U$. Conversely, if $\left|\begin{array}{ll}a & b \\ A & B\end{array}\right|=0$, it readily follows that $\Phi(a, A)=\Phi(b, B)$. We conclude the proof by showing that $\Phi$ is onto $\delta . \Phi(0,1)=\eta_{2} / \eta_{1}, \Phi(1,0)=I_{U}$, and

$$
\Phi(1,1)=\left(g_{3} I_{U}+g_{2} \eta_{2}\right) /\left(g_{3}+g_{2} \eta_{1}\right)
$$

are mutually distinct functions in $S$ since $\left|\begin{array}{ll}0 & 1 \\ 1 & 0\end{array}\right|,\left|\begin{array}{ll}1 & 1 \\ 0 & 1\end{array}\right|$, and $\left|\begin{array}{ll}0 & 1 \\ 1 & 1\end{array}\right|$ are all nonzero. For notational convenience let $F_{1}, F_{2}$, and $F_{3}$ denote $\Phi(0,1), \Phi(1,0)$, and $\Phi(1,1)$ respectively. Consider an arbitrary $F \in \mathcal{S}$. If $F=F_{1}$, then $\Phi(0,1)=F$. If $F \neq F_{1}$, then we shall show that $\Phi(1, A-1)=F$, where $A$ is defined by

$$
A=\frac{F_{1}-F_{2}}{F_{3}-F_{2}} \cdot \frac{F_{3}-F}{F_{1}-F} .
$$

Let $R\left(z_{1}, z_{2}, z_{3}, z_{4}\right)$ denote the cross-product

$$
\frac{z_{1}-z_{2}}{z_{3}-z_{2}} \cdot \frac{z_{3}-z_{4}}{z_{1}-z_{4}} \cdot
$$

We recall the elementary fact that the cross-product is invariant 
under the linear fractional transformation, i.e.,

$$
R\left[T\left(z_{1}\right), T\left(z_{2}\right), T\left(z_{3}\right), T\left(z_{4}\right)\right]=R\left(z_{1}, z_{2}, z_{3}, z_{4}\right) .
$$

We show that $A \in$ a. $A$ is meromorphic on $U$ since it is the ratio of meromorphic functions whose denominator is not identically zero.

Let $T \in M$, then for $\tau \in U$

$$
\begin{aligned}
A[T(\tau)] & =R\left\{F_{1}[T(\tau)], F_{2}[T(\tau)], F_{3}[T(\tau)], F[T(\tau)]\right\} \\
& =R\left\{T\left[F_{1}(\tau)\right], T\left[F_{2}(\tau)\right], T\left[F_{3}(\tau)\right], T[F(\tau)]\right\} \\
& =R\left[F_{1}(\tau), F_{2}(\tau), F_{3}(\tau), F(\tau)\right]=A(\tau) .
\end{aligned}
$$

Therefore, $A \in$ a. Solving (3) for $F$ we have

$$
F=\frac{F_{2}\left(F_{1}-F_{3}\right)+(A-1) F_{1}\left(F_{2}-F_{3}\right)}{\left(F_{1}-F_{3}\right)+(A-1)\left(F_{2}-F_{3}\right)} .
$$

Using the definitions of $F_{1}, F_{2}$, and $F_{3}$ this becomes

$$
F=\frac{g_{3} I_{U}+g_{2}(A-1) \eta_{2}}{g_{3}+g_{2}(A-1) \eta_{1}} \cdot \text { Q.E.D. }
$$

It follows immediately that $\delta$ has the power of the continuum since, for example, $\left|\begin{array}{ll}a & b \\ 1 & 1\end{array}\right|=0$ if and only if $a=b$.

4. An application. In this section we give an application of the functions in $\delta$. Two points $\tau, \tau^{\prime} \in U$ are said to be equivalent relative to $M$ if there exists a $T \in M$ such that $\tau^{\prime}=T(\tau)$. Two pairs $\left(\omega_{1}, \omega_{2}\right)$, $\left(\omega_{1}^{\prime}, \omega_{2}^{\prime}\right)$ are said to be equivalent if $\omega_{1}^{\prime}=\sigma\left(a \omega_{1}+b \omega_{2}\right), \omega_{2}^{\prime}=\sigma\left(c \omega_{1}+d \omega_{2}\right)$, where $\sigma \in C-\{0\}$ and $a, b, c, d$ are real integers satisfying $|a d-b c|$ $=1$.

THEOREM 2. Let $f$ be a pseudoperiodic function with pseudoperiods $H(\omega \mid \Omega)$ that satisfies (a) and (b). Further, let $f$ be such that

(c) $F$ defined by $F(\tau)=H\left(\tau \mid \Omega_{\tau}\right) / H\left(1 \mid \Omega_{\tau}\right), \tau \in U$, is not the identity function restricted to $U$.

(d) The set $\left\{\tau: H\left(\tau \mid \Omega_{\tau}\right)=\infty\right.$ or $H\left(1 \mid \Omega_{\tau}\right)=\infty$ or $\left.H\left(1 \mid \Omega_{\tau}\right)=H\left(\tau \mid \Omega_{\tau}\right)=0\right\}$ contains only finitely many mutually nonequivalent $\tau$ in $U$.

Then given $z_{1}, z_{2} \in C$ such that $\left|z_{1}\right|+\left|z_{2}\right|>0$, there exist $\omega_{1}, \omega_{2} \in C$, $\operatorname{Im} \omega_{1}^{-1} \omega_{2} \neq 0$, such that $H\left(\omega_{1} \mid \Omega\right)=z_{1}$ and $H\left(\omega_{2} \mid \Omega\right)=z_{2}$, where $\Omega=\Omega\left(\omega_{1}, \omega_{2}\right)$. Further, there exist infinitely many nonequivalent such pairs $\left(\omega_{1}, \omega_{2}\right)$.

Proof. We may assume that $z_{2} \neq 0$. Since $F \in S-\left\{I_{U}\right\}, F$ takes on every value of $\hat{C}$ at infinitely many points mutually nonequivalent relative to $M$ (see [4]). Hence, there exists a $\tau \in U$ such that 
$H\left(\tau \mid \Omega_{\tau}\right) / H\left(1 \mid \Omega_{\tau}\right)=z_{2} / z_{1} \neq 0$. By (d) we may assume that $H\left(\tau \mid \Omega_{\tau}\right) \neq 0, \infty$. Let $\lambda$ be a $(1 / \mu)$ th root of $z_{2} / H\left(\tau \mid \Omega_{\tau}\right)$ and let $\omega_{1}=\lambda, \omega_{2}=\lambda \tau$, $\Omega=\Omega\left(\omega_{1}, \omega_{2}\right)$. Then $H\left(\omega_{1} \mid \Omega\right)=H[\lambda \mid \Omega(\lambda, \lambda \tau)]=\lambda^{\mu} H\left(1 \mid \Omega_{\tau}\right)=z_{2} / H\left(\tau \mid \Omega_{\tau}\right)$ - $H\left(1 \mid \Omega_{\tau}\right)=z_{1}$. Similarly, $H\left(\omega_{2} \mid \Omega\right)=z_{2}$. To see that there exist infinitely many nonequivalent such pairs $\left(\omega_{1}, \omega_{2}\right)$, note that $\omega_{2} / \omega_{1}=\lambda \tau / \lambda$ $=\tau$ and that there exist infinitely many nonequivalent $\tau \in U$ such that $H\left(\tau \mid \Omega_{\tau}\right) / H\left(1 \mid \Omega_{\tau}\right)=z_{2} / z_{1} \neq 0$ and $H\left(\tau \mid \Omega_{\tau}\right) \neq 0, \infty$. Q.E.D.

We shall now exhibit a sequence of pseudoperiodic functions that satisfy (a)-(d). Let $N$ denote the set of nonnegative integers and let $P e: C \times \& \rightarrow \hat{C}$ denote the Weierstrass $P e$-function defined by

$$
P e(z \mid \Omega)=\frac{1}{z^{2}}+\sum_{\omega \in \Omega ; \omega \neq 0}\left[\frac{1}{(z-\omega)}-\frac{1}{\omega^{2}}\right] .
$$

For $n \in N$ let $Q_{n}: C \times \& \rightarrow \hat{C}$ denote the primitive of $[P e]^{n}$ (as a function of $z$ ) whose Laurent expansion around zero does not contain a constant term. It is shown in [1] that $Q_{n}$, for $n \in N-\{0,2\}$, satisfy (a)-(d). We observe that $Q_{1}=-\zeta$. Hence, in particular, there exist $\omega_{1}, \omega_{2}$, Im $\omega_{1}^{-1} \omega_{2} \neq 0$, such that $\eta\left(\omega_{1} \mid \Omega\right)=0$. This special case was considered by Watson [6] and Heins [4], among others.

5. A generalization. In this section we consider a natural generalization of the system of functional equations (1). Let $G$ be a Fuchsian group [2] with domain $D$ and consider the functions $F$ meromorphic on $D$ such that

$$
F[T(z)]=T[F(z)],
$$

for all $T \in G, z \in D$. Let $\oint_{G}$ denote the class of all such functions, and let $a_{G}$ denote the class of functions meromorphic on $D$ that are automorphic relative to $G$. The identity function on $D$ is in $\delta_{G}$, and it follows from $\$ 3$ that if $\delta_{G}$ contains three mutually distinct members $F_{1}, F_{2}$, and $F_{3}$, then

$$
F_{A}=\frac{F_{2}\left(F_{1}-F_{3}\right)+(A-1) F_{1}\left(F_{2}-F_{3}\right)}{\left(F_{1}-F_{3}\right)+(A-1)\left(F_{2}-F_{3}\right)}
$$

is in $S_{G}$ for all $A \in Q_{G}$. Further, $F_{A}=F_{B}$ if and only if $A=B$, and if $F \in \mathcal{S}_{G}-\left\{F_{1}\right\}$, then there exists an $A \in Q_{G}$ such that $F=F_{A}$. For the general Fuchsian group $G$ the author knows of no results concerning the existence, or nonexistence, of functions in $\delta_{G}-\left\{I_{D}\right\}$. In fact, the case where $G$ is an arbitrary finite group of linear fractional transformations and $S_{G}$ is a set of rational functions of $z$ appears to be unsettled. 


\section{REFERENCES}

1. M. M. Brady, Meromorphic solutions of a system of functional equations involving the modular group, Doctoral Dissertation, University of Illinois, Urbana, Ill., 1970.

2. L. Ford, Automorphic functions, McGraw-Hill, New York, 1929.

3. M. Heins, On the pseudoperiods of the Weierstrass zeta-functions, Siam J. Numer. Anal. 3 (1966), 266-268. MR 34 \#4548.

4. - - On the pseudoperiods of the Weierstrass zeta-functions. II, Nagoya Math. J. 30 (1967).

5. S. Saks and A. Zygmund, Analytic functions, PWN, Warsaw, 1965. MR 31 \#4889.

6. G. N. Watson, Periodic sigma functions, Proc. London Math. Soc. (3) 2 (1952), 129-149. MR 14, 43.

Esso Production Research Company, Houston, Texas 77001 